\title{
Cost-effectiveness of nurse-delivered cognitive behavioural therapy (CBT) compared to supportive listening (SL) for adjustment to multiple sclerosis
}

\author{
I. Mosweu ${ }^{1 *}$, R. Moss-Morris², L. Dennison ${ }^{3}$, T. Chalder $^{4}$ and P. McCrone ${ }^{1}$
}

\begin{abstract}
Background: Cognitive Behavioural Therapy (CBT) reduces distress in multiple sclerosis, and helps manage adjustment, but cost-effectiveness evidence is lacking.

Methods: An economic evaluation was conducted within a multi-centre trial. 94 patients were randomised to either eight sessions of nurse-led CBT or supportive listening (SL). Costs were calculated from the health, social and indirect care perspectives, and combined with additional quality-adjusted life years (QALY) or improvement on the GHQ-12 score, to explore cost-effectiveness at 12 months.

Results: CBT had higher mean health costs ( $£ 1610,95 \% \mathrm{Cl},-£ 187$ to 3771$)$ and slightly better QALYs $(0.0053,95 \%$ $\mathrm{Cl},-0.059$ to 0.103$)$ compared to SL but these differences were not statistically significant. This yielded $£ 301,509$ per QALY improvement, indicating that CBT is not cost-effective according to established UK NHS thresholds. The extra cost per patient improvement on the GHQ-12 scale was $£ 821$ from the same perspective. Using a $£ 20,000$, threshold, CBT in this format has a 9\% probability of being cost effective. Although subgroup analysis of patients with clinical levels of distress at baseline showed an improvement in the position of CBT compared to SL, CBT was still not cost-effective.

Conclusion: Nurse delivered CBT is more effective in reducing distress among MS patients compared to SL, but is highly unlikely to be cost-effective using a preference-based measure of health (EQ-5D). Results from a diseasespecific measure (GHQ-12) produced comparatively lower Incremental Cost-Effectiveness Ratios, but there is currently no acceptable willingness-to-pay threshold for this measure to guide decision-making.
\end{abstract}

Keywords: Multiple sclerosis, Cost-effectiveness, Costs, Cognitive behavioural therapy, Distress, Anxiety

\section{Background}

The clinical effects of multiple sclerosis (MS) and subsequent ambulatory complications associated with managing the condition may have psychological effects, which may not necessarily be treated with drugs. MS patients report very high levels of emotional distress and levels of major depression as high as $20 \%$ annually and $50 \%$ over a lifetime [1-3]. Depression in MS patients has

\footnotetext{
* Correspondence: iris.mosweu@kcl.ac.uk

'King's Health Economics, Institute of Psychiatry, Psychology \& Neuroscience, King's College London, Box 024, The David Goldberg Centre, De Crespigny Park, Denmark Hill, London SE5 8AF, UK

Full list of author information is available at the end of the article
}

been linked to disability, negative effects on quality of life, self-harming tendencies, as well as low adherence, which may eventually result in the discontinuation of disease modifying therapies (DMTs) $[4,5]$. However, elevated levels of distress or comorbid psychological disorders in MS are often overlooked in treatment [6]. There are particularly stressful moments in the trajectory of the illness (such as MS diagnosis, relapse, disease progression) and other critical life changing events when elevated levels of distress are to be expected [7].

Treating depression appears to improve adherence to Interferon beta-1B [8], and cognitive behavioural therapy (CBT) is increasingly being used to manage symptoms 
and enhance psychosocial outcomes for people with chronic conditions $[7,9,10]$. In MS, there is evidence that CBT may be helpful in reducing depression, anxiety, fatigue, disability, problems in dealing with cognition in addition to improving quality of life [7, 10-14]. A recent meta-analysis suggested psychological interventions (most of which were CBT-based) were at least moderately effective in treating depression in MS [14]. To date only one trial [15] has reported on the cost-effectiveness of a psychological adjustment group therapy for MS, and due to the significant amount of missing data in the quality of life data, quality-adjusted-life-years (QALYs) were not estimated, and the Beck Depression InventoryII was used to report a cost per point reduction on the scale of $£ 118$. Two pilot studies showed that web based CBT and SKYPE delivered mindfulness groups [16] may have the potential to be cost-effective in reducing fatigue and distress in MS respectively. However, these studies had no long-term follow up and data are preliminary.

A randomised controlled trial (RCT) of a nurse-led cognitive behavioural therapy for adjusting in the early stages of multiple-sclerosis reported that CBT is more effective in reducing distress for MS patients compared to supportive listening (SL) [17] up to one year follow up. The gains for CBT were also significantly greater for those who had clinically significant levels of distress at baseline. This paper reports on a cost-effectiveness study nested within this trial. We explored both costeffectiveness and cost utility in the whole cohort. We also conducted a subgroup analysis of patients who entered the trial with clinically significant levels of distress.

\section{Methods}

\section{Design and setting}

The study was a two-arm, randomised, multicentre parallel-group controlled trial. Patients were recruited from MS centres in Hampshire and South London, and randomly assigned to receive either CBT or SL. Full details of the trial methods, intervention components and findings have been reported previously $[10,18]$.

\section{Interventions}

Both interventions were delivered by general nurses specifically trained to provide eight one-on-one sessions, over a 10-week period. The sessions were delivered as a combination of two face-to-face meetings and six telephone calls.

\section{Self-reported outcomes}

Patients completed detailed measures at baseline, 15 weeks (end of treatment), 6 and 12 months postrandomisation with 12 months being the primary outcome point. The primary outcome was adjustment (defined as psychological well-being) measured using the
General Health Questionnaire (GHQ-12) [19]. The GHQ-12 uses a Likert scoring method, where high scores indicate higher distress, and to allow for a meaningful cost-effectiveness analysis, the GHQ 12 score was reported as a change score, multiplied by -1 to get an inverted score in which higher figures denote better outcomes.

Quality of life was assessed using (EQ-5D-3L) [20]. EQ-5D-3L is a brief self-reported preference-based measure which considers five dimensions of health (mobility, self-care, usual activities, pain/discomfort and anxiety/depression) each consisting of three levels of functioning (e.g. the levels for the pain dimension are no pain, moderate pain and extreme pain). This measure produces a possible 243 distinct health states ranging from 11111 (full health) to 33333 (worst) [21]. Value sets estimated from general population based studies were applied to the health states to produce preference-based scores between 0 (worse health) and 1 (full health). The measure also has a visual analogue scale ranging from 0 (the worst health you can imagine) to 100 (the best health you can imagine). Quality-adjusted-life-years (QALYS) were derived from transformed EQ-5D-3L scores, using the area-under-the-curve method [22].

\section{Service use and costs}

Contacts with the CBT and SL interventions were centrally recorded. Self-reported six-month retrospective health and social services data were collected at baseline, six- and 12-month follow-up, using an adapted version of the Client Service Receipt Inventory (CSRI) [23]. To ensure we captured relevant services the CSRI was adapted based on available literature and expert guidance. Patients provided details of their retrospective use of medication, inpatient, out-patient appointments, laboratory tests and scans, emergency department, contact with community professionals (GPs, neurologists, etc.) and informal care including access to social welfare benefits.

Service use was combined with nationally applicable unit costs [24-26] to derive total costs. Costs were measured in UK prices $(£$ ) for $2008 / 09$, and given the oneyear time horizon, there was no need to discount either costs or effects. Interventions costs were estimated by multiplying the unit cost of nurses providing the intervention by the number of sessions attended. Informal carers are not paid for their support to patients but there is still a value to this time. Informal care cost was estimated using the unit cost of a local authority home care worker as a proxy value [24]. Productivity costs were estimated through the human capital approach, which involves applying national wage rates to days off work due to illness. 


\section{Statistical analysis}

Data were analysed using Stata 11 and patients were assessed using an intention-to-treat analysis. Missing costs and QALY data were imputed using a regression-based method adjusting for baseline variables costs, EQ-5D-3L scores, GHQ-12 score, and the type of MS. Non-parametric bootstrapping methods were used to account for non-normality in the distribution of cost data [27]. Outcomes and costs were presented as mean values with standard deviations, and compared between groups at baseline and 12months follow-up.

The economic evaluation was conducted from both a health and social care, and societal perspectives. If one intervention had lower costs and better outcomes than the comparator, then it would be considered 'dominant'. In the event of the intervention having higher costs and better outcomes, cost-effectiveness would be assessed using incremental cost-effectiveness ratios (ICERs).

Cost-effectiveness planes (CEP) and cost-effectiveness acceptability curves (CEAC) were created to address the uncertainty around points estimates of the ICERs. For the CEP plane, non-parametric bootstrapping was used to create a joint distribution of incremental costs and outcomes, and plotting these differences on a scatter plot (one axis represents incremental costs and the other incremental outcome), to show the probability of the intervention being (i) cost-saving and more effective, (ii) cost-saving and less effective, (iii) cost-increasing and more effective and (iv) cost-increasing and less effective. The probability of the intervention being cost-effective was explored through the use of cost-effectiveness acceptability curve (CEAC), which were created by calculating a series of net benefits for a range $(£ 0-£ 60,000)$ of plausible values defined as a decision maker's willingness to pay for an additional unit improvement of health outcomes (e.g. QALYs) [28].

\section{Sub-group analysis}

The concurrent trial found that patients who had clinically significant distress at baseline showed meaningfully greater benefits from CBT over SL than those who were not distressed [7]. We conducted a sub-group analysis exploring cost-effectiveness for the distressed only, i.e. patients who scored three and above on GHQ-12 using the GHQ 0011 scoring method [29].

\section{Results}

Descriptive and clinical characteristics of participants

48 participants were randomised to CBT and 48 to SL. Patient characteristics at baseline were broadly similar across groups (Table 1), except that the SL group had a slightly higher mean age (43.1 vs. 40.3$)$; were more distressed (GHQ-12), disabled (EDSS scores), with slightly lower quality of life scores (EQ-5D-3L). The proportion (23\%) reporting use of antidepressants was higher for the CBT group ( 25 vs. 22 ), and more than half (60.6\%) had clinically significant distress levels.

\section{Service use and costs}

There were minimal differences in service use between the two groups. Participants accessed a wide range of health and social care services (Table 2), but the most intensively used were the GP ( $84 \%$ at baseline) and MS nurse ( $82 \%$ at baseline), followed by neurologists (66\%). The proportion with GP contacts had decreased by 12 month follow-up for both groups. The percentage in contact with neurologists and MS nurses was reduced over the follow-up period, with more reductions evident in the SL group for neurologists. Most participants reported the use of at least one medication (particularly disease modifying therapies) in both groups at baseline. Physiotherapists and alternative therapists had consistently low use over the entire study period for both groups.

Despite low admissions (10\%), the CBT group had substantially more inpatient days at 12 months. More than

Table 1 Baseline characteristics of participants

\begin{tabular}{lll}
\hline & CBT $(n=48)$ & SL $(n=46)$ \\
& Mean(S.D.) & $43.1(10.1)$ \\
\hline Mean age in years (SD) & $40.3(8.6)$ & $30(65.2)$ \\
Number of female participants (\%) & $35(72.9)$ & $24(52.2)$ \\
Number of Married/cohabiting participants (\%) & $30(62.5)$ & $33(71.7)$ \\
Number of White British participants (\%) & $38(79.2)$ & $30(65.2)$ \\
Number of participants with A level or higher (\%) & $35(72.9)$ & $5.1(1.0)$ \\
Mean EDSS score (SD) & $4.9(1.4)$ & $16.4(6.8)$ \\
Mean GHQ score (SD) & $14.0(5.5)$ & $0.60(0.26)$ \\
Mean EQ-5D score (SD) & $0.66(0.22)$ & $10(22)$ \\
Number treated for depression in the past year (SD) & $12(25)$ & $37(77)$ \\
Number diagnosed with relapsing remitting MS (SD) & $36(78)$ \\
\hline
\end{tabular}




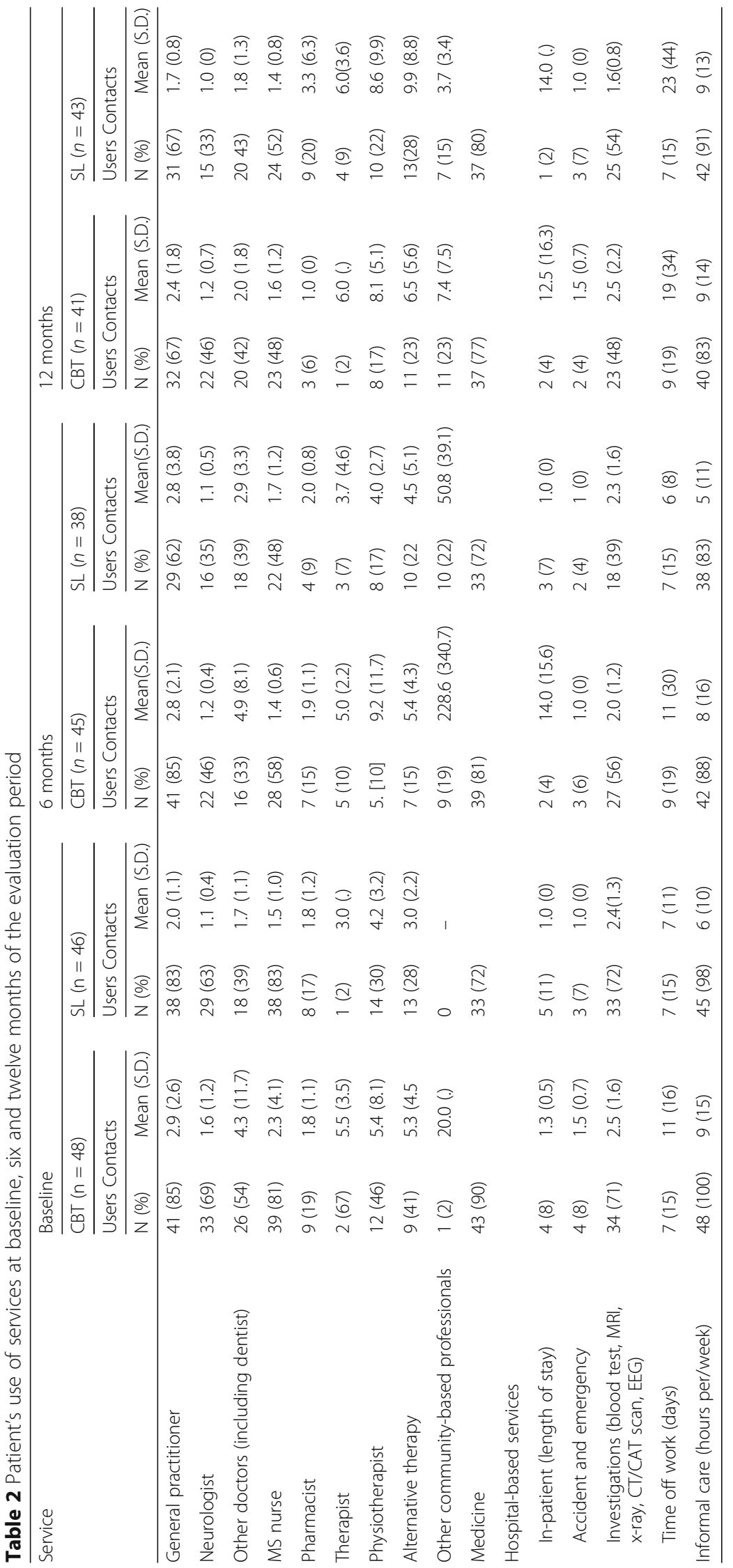


two thirds (71\%) reported investigations (e.g. blood tests, MRI, $x$-rays, CT/CAT and EEGs) at baseline, but at follow-up the proportions had decreased for both groups. The mean number of tests remained the same (2.5) for the CBT group, but had decreased (1.6) for the SL group. Almost all (99\%) patients reported informal care at baseline, and while this had decreased at followup, the mean hours/week were not so different. The mean cost of CBT was $£ 307$ compared to $£ 306$ for SL over 12 weeks. At baseline, mean service costs were fairly similar between the groups (Table 3 ), but higher informal care costs for the CBT group ( $£ 4378$ vs. $£ 2903$ ), contributed to much more societal costs at baseline compared to the SL group. Over the whole follow-up period, mean costs were higher in the CBT group. Drug costs contributed the highest share to health and social care costs while informal care added the greatest proportion to societal costs. Although the number receiving informal care were similar, the CBT group had greater intensity of use hence higher costs. From the health and social care (NHS) perspective CBT had higher mean costs at follow-up compared to SL ( $£ 7331$ vs. $£ 5026$ ), but this difference, adjusted for baseline costs $(£ 1610)$ was not statistically significant (bootstrapped $95 \% \mathrm{CI},-£ 187$ to 3771 ). The difference in mean costs from the societal perspective (£2871), was also not statistically significant (bootstrapped 95\% CI, $-£ 2028$ to $£ 7793)$.

\section{Cost-effectiveness analysis and cost-utility-analyses}

At baseline GHQ-12 scores were lower for the CBT group (13.98 vs. 16.40), and at 12 months both groups showed reductions in distress but the improvement in mean scores was better for the CBT group (2.69 vs. 1.97) and the difference(1.9572) statistically significant (bootstrapped 95\% CI,
-5.41 to -1.05$)$ [17]. Based on the change score, CBT produced an ICER of $£ 821 / G H Q-12$ score compared to SL from the health and social care perspective, indicating that for a one-point improvement on the GHQ-12 the NHS should pay $£ 821$. The ICER from the societal perspective is $£ 1242 / \mathrm{GHQ}-12$.

QALY results were also better for the intervention group at 12 months $(0.6627$ vs. 0.6197$)$ but the difference (0.0053) was not statistically significant (bootstrapped $95 \% \mathrm{CI},-0.059$ to 0.103$)$. CBT was therefore more effective at improving quality of life, but expensive, yielding an ICER of $£ 303,774$ from the health and social care perspective and $£ 541,698$ from the societal perspective.

\section{Subgroup analysis of those with clinical levels of distress at baseline}

Results from the subgroup analysis revealed a mean cost difference of $£ 1362$ (bootstrapped $95 \%$ CI, -781 to 3612) from the NHS perspective and $£ 3506$ (bootstrapped 95\% CI, -2704 to 9611 ) from the wider societal perspective. A statistically significant difference in the GHQ12 score (4.257 bootstrapped at 95\% CI, 1.109 to 7.521 ) produced a corresponding ICER of $£ 320$ from the NHS perspective and $£ 825$ from the societal perspective.

For the same analysis, a mean difference in QALYs of 0.0108 (bootstrapped 95\% CI, -0.051 to 0.068 ) indicates that the NHS would have to pay $£ 126,111$ for an additional QALY, while the society pays $£ 324,630$. Both these figures are higher than the UK cost-effectiveness threshold, but are substantially lower than the ICER in the base-case analysis.

The Cost-Effectiveness Plane (CEP) (Fig. 1) illustrates uncertainty around the estimated ICERs and it shows

Table 3 Summary of service costs and outcomes at baseline, six and twelve months

\begin{tabular}{|c|c|c|c|c|c|c|}
\hline \multirow{3}{*}{ Cost category } & \multicolumn{3}{|c|}{ Treatment $A(C B T) n=48$} & \multicolumn{3}{|c|}{ Treatment B (Supportive listening) $n=46$} \\
\hline & baseline & 6 months & 12 months & baseline & 6 months & 12 months \\
\hline & Mean (S.D) & Mean (S.D) & Mean (S.D) & Mean (S.D) & Mean (S.D) & Mean (S.D) \\
\hline Intervention & 0 & $307(170)$ & 0 & 0 & $306(148)$ & 0 \\
\hline Medication (drugs) & 2079 (1938) & $2724(3427)$ & $2531(4656)$ & $1928(2373)$ & $1476(2094)$ & $1882(2644)$ \\
\hline Community services (contacts with professionals) & $657(1130)$ & $422(430)$ & $483(715)$ & $466(402)$ & $305(331)$ & $395(482)$ \\
\hline Hospital services & $185(559)$ & $133(692)$ & $204(956)$ & $96(340)$ & $91(393)$ & $119(466)$ \\
\hline Investigations & $79(148)$ & $35(77)$ & $33(78)$ & $78(123)$ & $20(60)$ & $15(43)$ \\
\hline Total hospital and social care costs & 3000 (2098) & $3621(3751)$ & $3251(5134)$ & $2568(2295)$ & $2198(2507)$ & $2412(2654)$ \\
\hline Informal care & $4378(6970)$ & $3807(7463)$ & $4162(6352)$ & $2903(4550)$ & $2192(4993)$ & $4064(6242)$ \\
\hline Productivity loss & $1096(2726)$ & $704(3151)$ & $388(1742)$ & $719(2471)$ & $147(428)$ & 385 (1987) \\
\hline Total societal costs & $8473(8212)$ & 8132 (9036) & $7802(8819)$ & $6190(6183)$ & $4538(5605)$ & $6862(6922)$ \\
\hline Social benefits & $1210(1282)$ & $1430(1320)$ & $1229(1349)$ & $1325(1259)$ & $1360(1448)$ & $1640(1562)$ \\
\hline EQ-5D score & $0.659(0.215)$ & $0.661(0.216)$ & $0.644(0.267)$ & $0.595(0.262)$ & $0.641(0.198)$ & $0.622(0.274)$ \\
\hline GHQ-12 & $13.977(5.449)$ & & $11.289(4.63)$ & $16.391(6.771)$ & & $14.422(7.316)$ \\
\hline
\end{tabular}




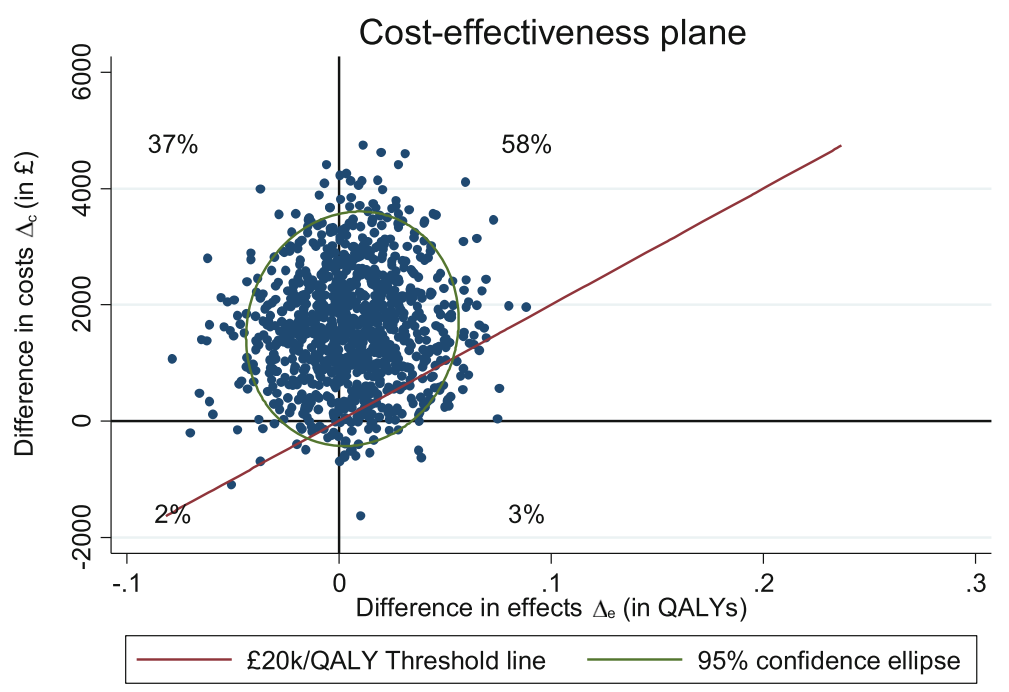

Fig. 1 Cost-effectiveness plane of CBT compared to SL using QALYS, adjusted for baseline costs and utility, from the NHS perspective

most $(58 \%)$ of the scatter points fall on the north-east quadrant which implies that CBT produces higher costs and better QALYs. The $£ 20,000$ threshold line has very few points below it, indicating that the probability of cost-effectiveness is likely to be very low at that willingness-to-pay threshold. The CEAC (Fig. 2) demonstrates the probability of the intervention being costeffective at varying willingness-to-pay thresholds per improvement in QALYs. Adjusted for baseline costs and utility scores, the CEAC indicates a $9 \%$ probability of CBT being cost-effective at $£ 20,000 / Q A L Y$ from the health and social care perspective, which confirms the CEP findings. The second CEP (Fig. 3) produced using the GHQ-12 score and cost from a similar perspective, however show $91 \%$ of the bootstrapped ICERs falling on the north-east quadrant, indicating that CBT is better at

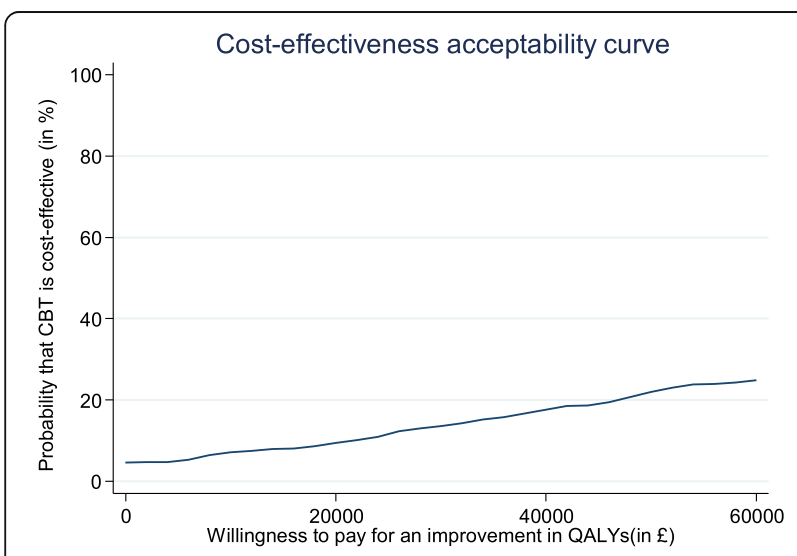

Fig. 2 Cost-effectiveness acceptability curve of CBT compared to SL, adjusted for baseline costs and utility, from the NHS perspective reducing psychological distress, albeit at higher costs, compared to SL.

\section{Discussion}

We have established that nurse-led CBT produces slightly better QALYs and is more effective in reducing psychological distress for MS, compared to SL. However, when the small effects are combined with incremental costs, CBT is not considered cost-effective compared to SL, according NICE guidelines. This conclusion was drawn from results of the ICER based on the EQ-5D-3L which show a cost per QALY of $£ 303,774$ from the health and social care perspective and $£ 541,698$ from the societal perspective. Sub-group analysis of patients meeting thresholds for clinical distress at baseline indicates a large improvement in the ICER $(£ 126,111)$ from the NHS and societal $(£ 324,629)$ perspectives, but still substantially higher than the recommended threshold in the UK. Results of the CEAC are also consistent with the ICER, showing only a $9 \%$ probability for CBT being cost-effective at the $£ 20,000$ threshold when compared with SL. Comparatively, the GHQ-12 results produced much lower ICERs from both the health and social care (£821), and the societal perspective (£1242). These figures are even lower when only the distressed group is analysed; $£ 320 / \mathrm{GHQ}-12$ from the NHS perspective and $£ 825$ from the societal perspective.

The difference in the ICERs is mainly driven by incremental differences in the outcome measures, whereupon the GHQ-12 detected sizeable differences and the EQ$5 \mathrm{D}-3 \mathrm{~L}$ produced very minimal differences. This could point to the insensitiveness of the EQ-5D-3L in patients with MS for all the five domains as illustrated in other previous work [30-32]. It is also possible that the EQ- 


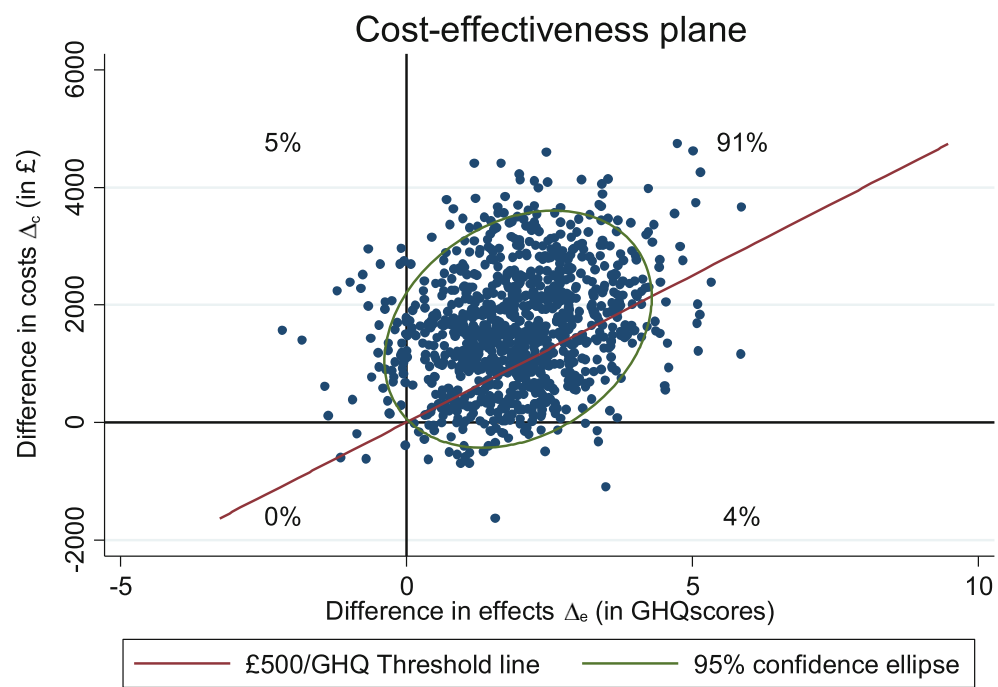

Fig. 3 Cost-effectiveness plane using GHQ-12 change scores for CBT compared to SL, adjusted for baseline GHQ-12 score and costs, from the NHS perspective

$5 \mathrm{D}-3 \mathrm{~L}$ is unable to detect the treatment effects of psychological interventions in MS patients. This is substantiated by previous work [15], in which the EQ-5D-3L failed to detect an effect in mood for MS patients. The sample size was estimated based on the primary clinical outcome, and it is possible that it was underpowered to demonstrate a significant difference in cost-effectiveness.

These results can assist in guiding researchers and policy-makers on areas to prioritise when considering interventions for MS patients.

As far as we know, this is the first economic evaluation of CBT for adjusting to multiple sclerosis. Whilst CBT has been found to be cost-effective in other disease areas such as depression [33], our results indicate that investing in nurse-led CBT may not proffer value for money compared to the same number of sessions of nurse-led SL for MS patients.

More work needs to be done to explore the potential cost-effectiveness of CBT in MS. Providing booster sessions at follow up may improve quality of life gains as the treatment effect for CBT at the end of treatment was greater than at follow-up [17]. It is also possible that there are subgroups within MS patients that could be targeted for future trials. It may be that future clinical trials target the therapy to those who need it most, like people with less social support or those screened and found to be distressed. Although our subgroup analysis did not produce costeffective results, other studies [15] reported clinical and cost-effectiveness of group psychological therapy for MS patients with low mood. The control group in Humphreys et al. [15], unlike ours, received usual care (without psychological interventions), which is a closer reflection of the current reality. Most patients with MS do not receive formal therapy for their distress so it may be that compared to treatment as usual, SL or CBT could be cost effective options. More work is needed to test this hypothesis.

\section{Limitations}

Limitations of the trial are outlined in our clinical paper [17], but the economic study had its own specific challenges. As is common in economic evaluations, there is a possibility of recall bias as participants reported on six months' retrospective service use. There is ongoing debate relating to the appropriateness of collecting resource use data, and for this trial the most pragmatic method was self-report and the recall period was determined by the trial design. There is currently no societal value linked to a unit improvement on the GHQ-12 score, making it hard to advice on the cost-effectiveness of CBT using this measure.

We used data from a clinical trial for our economic analysis. Sample size calculations in trials are generally based on the primary clinical outcome and not costs. However, most economic evaluations (including this one) focus on probabilities rather than testing for statistical significance in cost-effectiveness. Having said that, small samples do mean that we need to be cautious in our interpretation of findings and this small sample size is a limitation. We have referred to this in the discussion.

\section{Conclusion}

Nurse-led CBT compared to SL is not cost-effective for adjustment to MS using EQ-5D-3L but produces reasonable ICERs using GHQ-12. However, there is currently no acceptable willingness-to-pay threshold for this measure to guide in decision-making. 


\section{Implications for health care provision and use, health policies, and future research}

Further research in this area could be directed at designing CBT trials targeted to those who need it most, such as people with distress, as well as using alternative utility measures validated for psychological interventions in MS patients. We also know little about the costeffectiveness of different levels of clinical expertise. In this study, senior nurses who received a two-month training course provided the CBT. It is possible that experienced clinical psychologists, although costlier, may facilitate larger treatment effects and cost savings. Finally, recent evidence [34] suggests online therapy for depression may be an effective treatment in MS. Providing minimal therapy support alongside online therapy may be a cost-effective solution.

\section{Abbreviations \\ CBT: Cognitive Behavioural Therapy; CEAC: Cost-Effectiveness Acceptability Curve; CEP: Cost-Effectiveness Plane; CSRI: Client Service Receipt Inventory; CT: Computerised Tomography; DMT: Disease Modifying Therapy; EDSS: Expanded Disability Status Scale; EEG: Electroencephalogram; EQ- 5D: EuroQol Five Dimensions; GHQ-12: General Health Questionnaire; GP: General Practitioner; ICER: Incremental Cost-Effectiveness Ratio; MRI: Magnetic Resonance Imaging; MS: Multiple sclerosis; NHS: National Health Service; NICE: National Institute for Health and Care Excellence; QALY: Quality-Adjusted-Life-Year; RCT: Randomised Controlled Trial; SL: Supportive Listening}

\section{Acknowledgements}

Open access for this article was funded by King's College London.

\section{Funding}

This paper summarises independent research funded by the MS Society in the UK (award number 872/07). The views expressed in this publication are those of the author(s) and not necessarily those of the NHS, the National Institute for Health Research or the Department of Health. Trial registration: Current Controlled Trials ISRCTN91377356.

\section{Availability of data and materials}

The data that support the findings of this study are not available due to them containing information that could compromise research participant anonymity, and lack of permission from participants for the data to be passed on.

\section{Authors' contributions}

All authors meet the criteria for authorship in accordance with HER having made: a) substantial contributions to the conception or design of the study; or the data acquisition, analysis, or interpretation of data; b) drafting the manuscript or revising it critically for important intellectual content; c) final approval of the version to be published; and d) agree to be accountable for all aspects of the work in ensuring that questions related to the accuracy or integrity of any part of the work are appropriately investigated and resolved. IM is guarantor for the manuscript and data contained within. IM affirms that the manuscript is an honest, accurate, and transparent account of the study being reported; that no important aspects of the study have been omitted.

\section{Ethics approval and consent to participate}

Full informed consent was obtained before entry into the study. Ethical approval was obtained from the Thames Valley Multi Centre Research Ethics Committee (Ref: 07/MRE12/6).

\section{Consent for publication}

Not applicable.

\section{Competing interests}

The authors declare that they have no competing interests.

\section{Publisher's Note}

Springer Nature remains neutral with regard to jurisdictional claims in published maps and institutional affiliations.

\section{Author details \\ 'King's Health Economics, Institute of Psychiatry, Psychology \& Neuroscience, King's College London, Box 024, The David Goldberg Centre, De Crespigny Park, Denmark Hill, London SE5 8AF, UK. 'Department of Psychology, Institute of Psychiatry Psychology \& Neuroscience, King's College London, London, UK. ${ }^{3}$ School of Psychology, University of Southampton, Southampton, UK. ${ }^{4}$ Department of Psychological Medicine, King's College London, Cutcombe Road, London SE5 9RJ, UK.}

Received: 18 April 2017 Accepted: 22 September 2017

Published online: 10 October 2017

\section{References}

1. Patten SB, Beck CA, Williams JV, Barbui C, Metz LM. Major depression in multiple sclerosis: a population-based perspective. Neurology. 2003;61(11): $1524-7$.

2. Siegert R, Abernethy D. Depression in multiple sclerosis: a review. J Neurol Neurosurg Psychiatry. 2005:76(4):469-75.

3. Kern S, Schrempf W, Schneider H, Schultheiss T, Reichmann H, Ziemssen T. Neurological disability, psychological distress, and health-related quality of life in MS patients within the first three years after diagnosis. Mult Scler. 2009:15(6):752-8.

4. Patti F, Amato M, Trojano M, Bastianello S, Tola M, Picconi O, et al. Quality of life, depression and fatigue in mildly disabled patients with relapsingremitting multiple sclerosis receiving subcutaneous interferon beta-1a: $3-$ year results from the COGIMUS (COGnitive Impairment in MUltiple Sclerosis) study. Mult Scler J. 2011;17(8):991-1001.

5. Feinstein A. Multiple sclerosis, depression, and suicide. British medical Journal. 1997;BMJ. 315:691(0959-8138 (Print)).

6. Simpson R, McLean G, Guthrie B, Mair F, Mercer S. Physical and mental health comorbidity is common in people with multiple sclerosis: nationally representative cross-sectional population database analysis. BMC Neurol. 2014;14(1):128.

7. Dennison L, Moss-Morris R. Cognitive-behavioral therapy: what benefits can it offer people with multiple sclerosis? Expert Rev Neurother. 2010;10(9): 1383-90.

8. Mohr DC, Goodkin DE, Likosky W, Gatto N, Baumann KA, Rudick RA. Treatment of depression improves adherence to interferon beta-1 $\mathrm{b}$ therapy for multiple sclerosis. Arch Neurol. 1997;54(5):531-3.

9. Skokou M, Soubasi E, Gourzis P. Depression in Multiple Sclerosis: A Review of Assessment and Treatment Approaches in Adult and Pediatric Populations. ISRN Neurology. 2012;2012:6.

10. Moss-Morris R, Dennison L, Landau S, Yardley L, Silber E, Chalder T. A randomized controlled trial of cognitive behavioral therapy (CBT) for adjusting to multiple sclerosis (the saMS trial): does CBT work and for whom does it work? J Consult Clin Psychol. 2013;81(2):251-62.

11. Mohr DC, Hart S, Vella L. Reduction in disability in a randomized controlled trial of telephone-administered cognitive-behavioral therapy. Health Psychol. 2007;26(5):554-63.

12. van Kessel K, Moss-Morris R, Willoughby E, Chalder T, Johnson MH, Robinson E. A randomized controlled trial of cognitive behavior therapy for multiple sclerosis fatigue. Psychosom Med. 2008;70(2):205-13.

13. Cosio D, Jin L, Siddique J, Mohr DC. The effect of telephone-administered cognitive-behavioral therapy on quality of life among patients with multiple sclerosis. Ann Behav Med. 2011;41(2):227-34.

14. Fiest KM, Walker JR, Bernstein CN, Graff LA, Zarychanski R, Abou-Setta AM, et al. Systematic review and meta-analysis of interventions for depression and anxiety in persons with multiple sclerosis. Multiple Sclerosis and Related Disorders. 2016;5:12-26.

15. Humphreys I, Drummond Ae Fau - Phillips C, Phillips C Fau - Lincoln NB, Lincoln NB. Cost-effectiveness of an adjustment group for people with multiple sclerosis and low mood: a randomized trial. Clinical rehabilitation. 2013;0(1477-0873 (Electronic)). 
16. Bogosian A, Chadwick P, Windgassen S, Norton S, McCrone P, Mosweu I, et al. Distress improves after mindfulness training for progressive MS: A pilot randomised trial. Multiple Sclerosis Journal.

17. Moss-Morris R, Dennison L, Landau S, Yardley L, Silber E, Chalder T. A Randomized Controlled Trial of Cognitive Behavioral Therapy (CBT) for Adjusting to Multiple Sclerosis (the saMS Trial): Does CBT Work and for Whom Does It Work? J Consult Clin Psychol. 2012;81(2):251-62.

18. Moss-Morris R, Dennison L, Yardley L, Landau S, Roche S, McCrone P, et al. Protocol for the saMS trial (supportive adjustment for multiple sclerosis): a randomized controlled trial comparing cognitive behavioral therapy to supportive listening for adjustment to multiple sclerosis. BMC Neurol. 2009;9:45.

19. Goldberg D. General health questionnaire (GHQ-12). Nfer-Nelson: Windsor, UK; 1992.

20. Williams A. The role of the EuroQol instrument in QALY calculations: Centre for Health. Economics Discussion Paper [Internet]. 1995; 130.

21. Rabin R, de Charro F. EQ-5D: a measure of health status from the EuroQol Group. Ann Med. 2001;33(5):337-43.

22. Brazier J, Ratcliffe J, Salomon J, Tsuchiya A. Measuring and Valuing Health Benefits for Economic Evaluation. Oxford: Oxford University Press; 2007.

23. Beecham J, Knapp M, editors. Costing psychiatric interventions, in Measuring Mental Health Needs. Gaskell: London; 2001.

24. Curtis $L$, editor. Unit Costs of Health and Social Care. : Personal Social Services Research Unit, University of Kent, 2008; 2008.

25. Department of Health. National Health Service Schedule of Reference Costs 2007/08 London2009 [Available from: http://webarchive.nationalarchives. gov.uk//http://www.dh.gov.uk/en/Publicationsandstatistics/Publications/ PublicationsPolicyAndGuidance/DH_095859.

26. British Medical Association RPS. British National Formulary, 49. London, 2008: RMJ Publishing, 2008.

27. Barber JA, Thompson SG. Analysis of cost data in randomized trials: an application of the non-parametric bootstrap. Stat Med. 2000;19(23):3219-36.

28. Fenwick E, Byford S. A guide to cost-effectiveness acceptability curves. The British journal of psychiatry : the journal of mental science. 2005;187:106-8.

29. Nicholl CR, Lincoln NB, Francis VM, Stephan TF. Assessment of emotional problems in people with multiple sclerosis. Clin Rehabil. 2001;15(6):657-68.

30. Jones KH FD, Jones PA, et al. How People with Multiple Sclerosis Rate Their Quality of Life: An EQ-5D Survey via the UK MS Register. Reindl M, ed. PLoS ONE. 2013;8(6):e65640. doi:https://doi.org/10.1371/journal.pone.0065640. PLOS ONE. 2013;8(6).

31. Svensson M, Fajutrao L. Costs of Formal and Informal Home Care and Quality of Life for Patients with Multiple Sclerosis in Sweden. Mult Scler Int. 2014;2014:7.

32. Kuspinar A, Mayo NE. Do generic utility measures capture what is important to the quality of life of people with multiple sclerosis? Health Qual Life Outcomes. 2013;11(1):71

33. Hollinghurst S, Carroll FE, Abel A, Campbell J, Garland A, Jerrom B, et al. Cost-effectiveness of cognitive-behavioural therapy as an adjunct to pharmacotherapy for treatment-resistant depression in primary care: economic evaluation of the CoBalT Trial. Br J Psychiatry. 2014;204(1):69-76.

34. Fischer A, Schröder J, Vettorazzi E, Wolf OT, Pöttgen J, Lau S, et al. An online programme to reduce depression in patients with multiple sclerosis: a randomised controlled trial. The Lancet Psychiatry. 2015;2(3):217-23.

\section{Submit your manuscript to a SpringerOpen ${ }^{\circ}$ journal and benefit from:}

- Convenient online submission

- Rigorous peer review

- Open access: articles freely available online

- High visibility within the field

- Retaining the copyright to your article

Submit your next manuscript at $\gg$ springeropen.com 